\title{
Perfil sociodemográfico e necessidades de educação em saúde entre cuidadores de idosos em uma unidade de saúde da família em llhéus, Bahia, Brasil
}

\author{
Sociodemographic profile and health education needs amongst caregivers of the elderly in a family \\ health unit in Ilhéus, Bahia, Brazil

\section{Perfil sociodemográfico y las necesidades de educación en salud entre los cuidadores de los ancianos en una unidad de salud familiar en Ilhéus, Bahia, Brasil}

\author{
Elaine Rodrigues Coelho. Universidade Estadual de Santa Cruz (UESC). Ilhéus, BA, Brasil. laneegbi@hotmail.com (Autora correspondente) \\ Daniel dos Santos Sacerdote. Universidade Estadual de Santa Cruz (UESC). Ilhéus, BA, Brasil. daniel_santos_7@hotmail.com \\ Laís Tailla Santos Cardoso. Universidade Estadual de Santa Cruz (UESC). Ilhéus, BA, Brasil. laistailla@bol.com.br \\ Rosana Maria de Carvalho Santos Barreto. Secretaria Municipal de Saúde de Ilhéus. Ilhéus, BA, Brasil. roca_barreto@hotmail.com \\ Rozemere Cardoso de Souza. Universidade Estadual de Santa Cruz (UESC). Ilhéus, BA, Brasil. rozemeresouza@hotmail.com
}

\section{Resumo}

Objetivos: Caracterizar o perfil sociodemográfico e possíveis necessidades de educação em saúde de cuidadores de idosos vinculados a uma Unidade de Saúde da Família (USF) em Ilhéus, Bahia. Métodos: Estudo transversal descritivo, realizado com 26 cuidadores de idosos. Os dados foram coletados entre os meses de agosto e setembro de 2011, a partir da aplicação de questionário semiestruturado. A análise dos dados foi feita a partir da distribuição de frequência, que permitiu conhecer o perfil dos cuidadores e suas necessidades de educação em saúde. Resultados: Verificou-se que, entre os cuidadores, a maioria era representada por mulheres entre 30 e 40 anos, donas de casa, com predominância do nível escolar fundamental incompleto. Mais da metade apresentava grau de parentesco consanguíneo com o idoso e não recebia pagamento por esse tipo de atividade. Quanto ao interesse em participar de intervenção educativa que enfatizasse temas do cuidado ao idoso, a maioria dos participantes respondeu afirmativamente à questão. Algumas das necessidades de educação em saúde identificadas foram: saber lidar com a instabilidade de humor do idoso, conhecer a alimentação mais adequada e superar os obstáculos para a mobilidade do ancião. Conclusão: 0 estudo oferece auxílio para a construção de uma linha de cuidados para o idoso e o cuidador, o planejamento de práticas educativas que preencham lacunas de conhecimento dos sujeitos investigados, contribuindo para a reflexão sobre processos educacionais que valorizem habilidades do cuidador.

\section{Abstract}

Objectives: To characterize the sociodemographic profile and possible health education needs of caregivers of elderly patients linked to a Family Healthcare Unit (FHU) in the municipality of Ilhéus, Bahia state, Brazil. Methods: Descriptive transversal study conducted with 26 caregivers of elderly patients. Data were collected through application of semi-structured questionnaires from August to September 2011. Data analysis was performed by frequency distribution, which provided understanding about the caregivers' profile and their needs in health education. Results: It was possible to verify that most caregivers were housewives with incomplete primary education between 30 and 40 years old. More than half presented a degree of consanguinity with the elderly relative they cared for and were not paid for the activity. Concerning their interest in participating in educational interventions that emphasize topics about elderly care, most participants answered the question affirmatively. Some of the needs in health education identified were as follows: knowing how to deal with elderly mood instability; knowing the most appropriate diet requirements; and overcoming obstacles to mobility. Conclusion: This study gives support to the construction of a line of care for both the elderly and their caregivers and the planning of educational interventions that narrow the knowledge gap of those researched, offering reflections on educational processes that value caregivers' skills.

Palavras-chave: Cuidadores Atenção Primária à Saúde Saúde do Idoso Educação em Saúde

Keywords: Caregivers Primary Health Care Health of the Elderly Health Education 


\section{Resumen}

Objetivo: Caracterizar el perfil socio-demográfico y las necesidades de educación en salud de cuidadores de anciano vinculados a una Unidad de Salud Familiar (USF) de Ilhéus, Bahia. Métodos: Estudio transversal descriptivo, realizado con 26 cuidadores de ancianos. Los datos fueron recogidos entre agosto y septiembre de 2011, a través de la aplicación de un cuestionario semi-estructurado. El análisis se realizó a partir de la distribución de frecuencia, lo que permitió conocer el perfil de los cuidadores y sus necesidades de educación en salud. Resultados: Se encontró que, la mayoría de los cuidadores eran mujeres de entre 30 y 40 años, amas de casa, principalmente con nivel de escolaridad primario incompleto. Más de la mitad tenía un vínculo de parentesco de primer grado con los ancianos y no recibía remuneración por esa tarea. La mayoría de los participantes respondió afirmativamente a la pregunta sobre el interés por participar en una intervención educativa sobre temas relacionados al cuidado de los ancianos. Algunas de las necesidades de aprendizaje identificadas fueron: saber lidiar con la inestabilidad del humor de los ancianos, conocer la alimentación más adecuada y superar los obstáculos a la movilidad de los ancianos. Conclusión: Este estudio ofrece soporte para la construcción de una línea de cuidados tanto para los ancianos como para sus cuidadores, para la planificación de prácticas educativas que cubran brechas en el conocimiento de los sujetos investigados, contribuyendo con las reflexiones sobre los procesos educativos que valoricen las habilidades del cuidador.
Palabras clave:

Cuidadores

Atención Primaria de la Salud

Salud del Anciano

Educación en Salud

\section{Introdução}

O envelhecimento - processo complexo, caracterizado por mudanças biológicas, sociais, psicológicas e culturais - é um fenômeno mundial, resultante, dentre outros aspectos, do desenvolvimento econômico, dos avanços científicos e da redução das desigualdades sociais. No Brasil, estima-se que, em 2050, a população idosa ultrapassará os $22,71 \%$ da população total $^{1}$. Apesar do rápido envelhecimento, percebe-se que a organização do sistema público de saúde ainda não conseguiu se desenvolver com a mesma rapidez ${ }^{2}$, situação que justifica trabalhos sobre a necessidade emergente de implantação de políticas públicas a fim de garantir qualidade, respeito e dignidade aos idosos e seus familiares.

O envelhecer também acarreta aumento das condiçóes crônicas de saúde, podendo haver perda progressiva da capacidade de adaptação ao ambiente e da autonomia, tornando o idoso dependente de auxílio temporário ou permanente de outra pessoa para realizaçáo das atividades da vida diária e de autocuidado ${ }^{3,4}$. Muitos idosos restritos em seus domicílios apresentam alto grau de dependência, decorrente das incapacidades funcionais e não têm acesso aos programas relacionados à terceira idade que visam à qualidade de sua vida e à interação social ${ }^{2}$.

Além das doenças crônicas que acometem os idosos, merecem destaque condiçôes patológicas relacionadas à deficiência auditiva ${ }^{5}$, aos traumas, à violência intrafamiliar ${ }^{6,7}$, depressão e às condiçóes sociodemográficas que fragilizam a saúde do idoso, a exemplo dos baixos níveis de escolaridade e de renda 8 .

Disso decorre o surgimento, cada vez maior, de novas e diferentes necessidades de cuidado ${ }^{4}$, e a importância de se ter como alvo, no âmbito das políticas públicas, reflexôes e açóes sobre o conhecimento, o planejamento e a execução desses modos de cuidar/produzir saúde e qualidade de vida do idoso na comunidade.

A Política Nacional de Saúde da Pessoa Idosa (PNSPI) prevê a importância da recuperação, manutenção e promoção da autonomia e da independência da pessoa idosa. Reconhece ainda, a probabilidade que idosos têm de sofrer algum tipo de doença crônica não transmissível (DCNT) ou de necessitar do auxílio de um cuidador, decorrente de uma doença limitadora? .

$\mathrm{Na}$ família, nem todos assumem o cuidado com o idoso da mesma maneira ${ }^{10}$. O cuidador principal - quase sempre um de seus membros - é definido como aquela pessoa responsável pelas necessidades de cuidado do idoso. Nomeiam-se cuidadores secundários os demais sujeitos - integrantes da família ou não - que surgem para contribuir, complementando esses cuidados ${ }^{3,11}$.

Para prezar e assegurar a saúde do idoso, como também a do cuidador, destaca-se a importância do conhecimento acerca do perfil do cuidador, bem como de suas lacunas de conhecimento durante a prática do cuidado. No entanto, os cuidadores nem sempre encontram oportunidades de ensino/aprendizagem sobre como cuidar do idoso na perspectiva de um envelhecimento saudável.

O fato de os cuidadores não apresentarem conhecimentos básicos sobre o cuidado do idoso agrava o desgaste familiar, podendo gerar conflitos internos, processos depressivos e outras doenças ${ }^{3}$.

A tarefa do cuidar é considerada um desafio 'crescente', visto que, com o avançar da idade, os idosos podem se tornar ainda mais dependentes. Os cuidadores, muitas vezes, não recebem orientação de um profissional de saúde no que diz respeito à prática do cuidado e realizam suas tarefas com o aprendizado adquirido pela experiência cotidiana advinda do 
ato de cuidar ${ }^{12}$. A Estratégia Saúde da Família (ESF) oferece cenário apropriado para permitir que o cuidador fortaleça o vínculo com o profissional de saúde junto às famílias, seja em visitas domiciliares ou em consultas, como meio de apoiar o cuidado integral ao idoso.

Para desenvolver uma futura intervenção educativa para os cuidadores de idosos em uma Unidade de Saúde da Família vinculada ao Programa de Educação pelo Trabalho para a Saúde (PET-Saúde) e no intuito de aproximar profissionais de saúde da família e cuidadores, este estudo teve por objetivo caracterizar o perfil sociodemográfico e possíveis necessidades de educação em saúde de cuidadores de idosos vinculados a uma Unidade de Saúde da Família no município de Ilhéus, Bahia.

\section{Métodos}

Trata-se de estudo transversal descritivo, realizado na área de abrangência de uma Unidade de Saúde da Família (USF) vinculada ao Programa de Educação pelo Trabalho para Saúde: PET-Saúde, na cidade de Ilhéus - BA. O PET-Saúde amplia a atuaçáo de estudantes nos serviços de saúde e contribui para integrar ensino, serviço e comunidade.

O estudo também faz parte de um projeto mais amplo, intitulado Processo de construçáo das linhas de cuidado em saúde no município de Ilhéus, Bahia, aprovado pelo Comitê de Ética em Pesquisa com seres humanos da Universidade Estadual de Santa Cruz (UESC), sob protocolo de número 568/2010.

Do total de 152 idosos cadastrados na referida unidade, foram identificados 26 (vinte e seis) cuidadores que participaram deste estudo por atenderem aos seguintes critérios de inclusão: cuidar de idoso cadastrado na área de abrangência da USF local; consentir a participação na pesquisa mediante assinatura do termo de consentimento livre e esclarecido dos sujeitos. Fez-se a identificação desses cuidadores por meio de levantamento junto aos Agentes Comunitários de Saúde (ACS) - aqui considerados informantes-chave. No total, 26 cuidadores, distribuídos entre três microáreas, aceitaram participar do estudo. Sendo assim, não houve registro de recusa. Em duas microáreas, os dados não foram coletados, pois estavam descobertas quanto à atividade do ACS.

Denominou-se cuidador de idoso toda a pessoa referida pelo idoso ou familiar como sendo o principal responsável por atender às necessidades de saúde do idoso, inclusive aquelas relacionadas a acesso aos serviços de saúde, em todos os níveis de complexidade da atenção, independente do grau de dependência do idoso. Sobre este último aspecto - grau de dependência do idoso - apesar da ausência de dados, pressupóe-se que a situação de vulnerabilidade social observada na maioria das áreas de abrangência da ESF caracterize condição que justifica a presença de cuidador. Associa-se a isto a vulnerabilidade de atenção programática da pessoa idosa, definida pela insuficiência de serviços diante das necessidades dessa população ${ }^{13}$. Ambas as situaçóes ainda estão presentes no território estudado, podendo elevar e/ou definir a dependência do idoso por um cuidador.

A coleta de dados dos cuidadores foi realizada entre os meses de agosto e setembro de 2011, mediante aplicação domiciliar de um questionário semiestruturado, construído a partir de leituras sobre o tema e de reunióes de equipe, sendo dividido em duas partes: perfil do cuidador (sexo, idade, profissão, escolaridade, parentesco com o idoso, condiçóes socioeconômicas e tempo dispensado ao cuidado) e educação em saúde (interesse em participar de uma intervenção educativa, temas de maior interesse, principais dificuldades durante o cuidado).

Para caracterização dos idosos sob os cuidados dos participantes investigados, utilizou-se a Ficha A - ficha de cadastramento das famílias -, instrumento de coleta de dados do Sistema de Informação da Atenção Básica (SIAB), com informaçóes sociodemográficas e clínicas atualizadas de todos os usuários da ESF. Por motivo de náo identificaçáo da referida ficha, dados de dois idosos foram excluídos deste estudo.

Para análise, os dados foram organizados em planilha Excel ${ }^{\bullet}$ versão 2007 e submetidos à distribuição de frequência simples, permitindo caracterizar o perfil dos cuidadores e suas possíveis necessidades de educação em saúde. Fez-se também o entrelaçamento dos resultados, articulando-os aos saberes que constam na literatura especializada sobre o tema. 


\section{Resultados}

A seguir, é apresentada uma breve caracterizaçáo dos idosos cuidados pelos participantes deste estudo, seguida pelos dados sobre o perfil sociodemográfico e necessidades de educação em saúde desses cuidadores.

\section{Caracterização dos idosos}

Entre os vinte e quatro idosos que dependiam dos cuidadores investigados neste estudo, os dados que constavam na ficha de cadastramento das famílias foram os seguintes: $62,50 \%$ deles eram do gênero feminino e 37,50\% do masculino, com idades variando entre 64 e 83 anos, com média de 74,12 anos (desvio padrão: 7,77). Quanto à escolaridade, a maioria dos idosos $(62,50 \%)$ era alfabetizada.

Os dados clínicos revelaram os seguintes diagnósticos/problemas de saúde: 11 idosos (45,80\%) com Hipertensão Arterial Sistêmica; 8 (33,30\%) eram portadores de Diabetes Mellitus; 3 (12,50\%) tinham Deficiência Física; 1 (4,20\%) era Alcoolista; e 1 (4,20\%) apresentava Deficiência Cognitiva. Em relação à pergunta presente na Ficha A, "Em caso de doença, a quem procura?", 17 (70,80\%) idosos possuíam fichas referindo procurar o hospital e apenas $7(29,20 \%)$ faziam referência à procura da USF para essa condição.

\section{Perfil sociodemográfico e necessidades de educação em saúde dos cuidadores}

No que tange ao perfil do cuidador, notou-se que $88 \%$ eram mulheres, donas de casa, na faixa etária entre 30 e 40 anos. Do total de 26 cuidadores, 10 (38\%) possuíam nível escolar fundamental incompleto e somente três indivíduos apresentavam nível superior completo. Os dados mostram que os cuidadores náo recebiam pagamento pelo cuidado desenvolvido, mas 58,00\% deles referiram renda individual menor que um salário mínimo - renda proveniente de outros serviços não especificados.

Mais da metade (73\%) dos entrevistados eram cuidadores informais e/ou integrantes da família, enquanto que os cuidadores formais (27\%) reconheciam-se como amigos do idoso e não eram integrantes da família. O percentual dos cuidadores identificados no presente estudo como familiares dos idosos está distribuído conforme relação de parentesco na Figura 1.

No que se refere ao tempo dispensado ao cuidado, $92 \%$ dos cuidadores ofereciam cuidado todos os dias da semana, mas desenvolviam suas atividades em tempos diários diferenciados, explicitados na Tabela 1. Vinte cuidadores alegaram nunca ter recebido nenhum tipo de treinamento para prestação do cuidado ao idoso, valendo-se de suas próprias experiências de vida. Associado a isto, ressalta-se que 38\% deles não recebiam ajuda de outra pessoa, sendo os únicos responsáveis pelo cuidado.

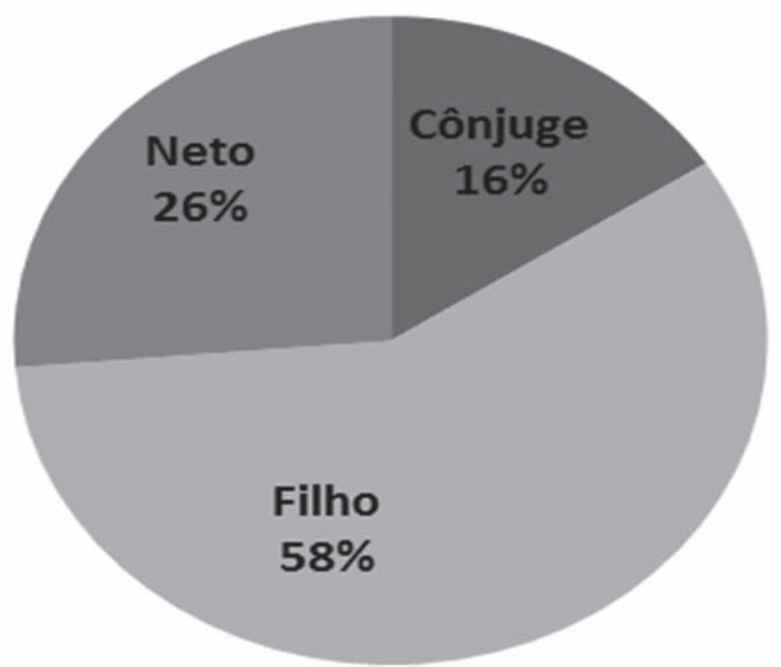

Figura 1. Distribuição da frequência de cuidadores de idosos quanto ao grau de parentesco consanguíneo com o idoso em Unidade de Saúde da Família no município de Ilhéus, Bahia, 2011. 
Tabela 1. Distribuição da frequência de cuidadores de idosos quanto ao tempo diário dedicado à prestação do cuidado aos idosos em USF no município de llhéus, Bahia, 2011.

\begin{tabular}{lcc}
\hline \multicolumn{1}{c}{ Tempo $(\mathrm{h}) /$ dia } & $\mathrm{N}$ & $\%$ \\
\hline Até 4 horas & 4 & 15 \\
5 a 8 horas & 3 & 12 \\
9 a 12 horas & 3 & 12 \\
Mais que 12 horas & 16 & 62 \\
Total & 26 & 100 \\
\hline
\end{tabular}

As dificuldades mais citadas pelos participantes da pesquisa no contexto do cuidado ao idoso estavam relacionadas às características psicossociais do idoso, entre elas, variaçáo do humor e resistência ao cuidado oferecido. $\mathrm{O}$ fato de o cuidador ser único, náo contar com qualquer tipo de apoio durante suas atividades, além da falta de tempo para cuidar de si próprio também foi mencionado, todavia com menor expressividade do que os outros obstáculos, como os listados na Tabela 2.

Tabela 2. Distribuição da frequência das dificuldades encontradas por cuidadores durante a prática de cuidado aos idosos em USF no município de llhéus, Bahia, 2011.

\begin{tabular}{lcc}
\hline \multicolumn{1}{c}{ Dificuldades referidas pelos cuidadores } & N & (\%) \\
\hline Realizar higiene/ dar banho & 6 & 12,30 \\
Deslocar-se com o idoso & 7 & 14,30 \\
Alimentação do idoso & 3 & 6,10 \\
Horários de medicamentos & 3 & 6,10 \\
Variação de humor do idoso & 11 & 22,40 \\
Cuidar no período noturno & 3 & 6,10 \\
Ser o único cuidador & 5 & 10,20 \\
Falta de tempo para cuidar de si próprio & 5 & 10,20 \\
Resistência do idoso ao cuidado & 6 & 12,30 \\
Total & 49 & 100 \\
\hline
\end{tabular}

Quando questionados sobre o desejo em participar de uma intervenção educativa, este foi evidenciado pela maioria dos participantes (88\%). Houve demonstração de interesse nas seguintes temáticas relacionadas ao idoso: cuidados psicológicos e emocionais (18,60\%); planejamento da administração de remédios (17,50\%); cuidados com a mobilidade e com a postura $(15,10 \%)$; alimentação $(15,10 \%)$; cuidados com a higiene corporal $(9,30 \%)$; e como realizar curativos $(9,30 \%)$. Em relação aos $12 \%$ que referiram não ter interesse em participar de uma intervenção educativa, embora questionados, não foram explicitadas as justificativas.

\section{Discussão}

A caracterização do perfil sociodemográfico dos cuidadores de idosos mostra um baixo nível de renda acompanhado de pouca escolaridade, resultado semelhante ao encontrado em outros estudos ${ }^{12,14-16}$, de modo que, a existência de associaçáo entre condições financeiras frágeis e baixa escolaridade constituem fortes preditores de tensão para o cuidador ${ }^{16}$. Além disso, “[...] essas condições sociais diminuem a participação efetiva na busca de implementação de soluçóes consistentes de proteção e elevação dos níveis de saúde do idoso.".

Nesse contexto, destaca-se o alto percentual de mulheres como cuidadoras (88\%), fenômeno universalmente observado $3,7,10,17,18$. Esse dado é gerador de reflexóes sobre a predominância do sexo feminino nesse grupo e suas implicaçóes: "[...] somadas àquelas de sua segunda jornada de trabalho, na qual assumem o papel de esposa, zeladora do lar e dos filhos, [e que] contribuem para que a sobrecarga de atividades torne-se extrema." ${ }^{17}$.

Por sua vez, a opção pela filha como cuidadora principal converge para expectativas de idosos em situação de independência quando questionados sobre quem gostariam que fosse seu futuro cuidador ${ }^{19}$. $\mathrm{Na}$ Bahia, essa escolha também foi evidenciada em estudo com cuidadores, pois $84,40 \%$ deles destacaram a preferência de ser cuidado na velhice por seus filhos e outros familiares com destaque para "[...] que seja com carinho e, sempre que possível, na sua própria casa."3. 
O estudo mostra aspectos que podem dificultar a atuação dos cuidadores na qualidade de sujeitos sociais. As questôes emocionais e psicossociais do idoso cuidado, como agressividade e resistência, sobressaem-se e apontam para a importância de intervençôes estratégicas à essa população, como forma de melhorar o desempenho do cuidador, com aumento de suas habilidades e controle sobre as açôes desenvolvidas diante das demandas exigidas para esse tipo de cuidado. O próprio envelhecer instiga o surgimento de mudanças psicológicas, as quais exigem habilidades e conhecimento durante o ato de cuidar e da convivência com os idosos ${ }^{20}$.

O entendimento das necessidades humanas e suas mudanças psicológicas, sociais e culturais oferecem subsídio a uma prática de cuidado integral ${ }^{21}$, no entanto, a carência de conhecimentos sobre o envelhecimento e a ausência de técnicas de cuidado por parte do cuidador, associadas a uma estrutura econômica que não oferece apoio ao idoso e sua família, dificultam uma assistência integral. Então, "[...] a dinâmica cuidador e idoso poderá sofrer interferência de emoçôes negativas e que tendem a resultar em potencial risco de violência.".

Além disso, outros fatores de risco ao cuidado integral descrevem-se por estresse/fadiga do familiar cuidador, relacionado ao fato de ser cuidador único e em tempo prolongado, e ao isolamento resultante da responsabilidade pelo cuidar e/ou ao grau de dependência do idoso. Tais fatores revelam fortes indícios de desencadeamento de violência intrafamiliar e exigem atençấo dos serviços sociais e de saúde para medidas preventivas e de apoio ao binômio idoso e família ${ }^{6}$.

O papel de cuidar, então, é um compromisso que pode ser penoso ou não, dependendo das circunstâncias envolvidas na relaçáo e do ambiente que as circunda ${ }^{6}$. Por exemplo, o cuidado prestado (quase sempre por mais de 8 horas por dia) gera sobrecarga e afeta negativamente a relação idoso-cuidador. A literatura descreve relatos de mágoas surgidas pelo desgaste físico e psíquico durante a tarefa do cuidar ${ }^{3,6,10,22-24}$.

Neste sentido, destacam-se as folgas, nomeadas de "respiro do cuidador", como uma forma de alívio da sobrecarga de cuidadores de idosos, previstas no relatório Mundial sobre a Saúde Mental, a fim de aprimorar a saúde dos provedores de saúde ${ }^{25}$.

O presente estudo possibilitou mostrar o interesse dos cuidadores em participar de uma atividade educativa - evidenciada no estudo de Souza et al. ${ }^{21}$, e suas principais necessidades de Educação em saúde: lidar com variação de humor, conhecer alimentação adequada e uso correto dos medicamentos. Intervir nesse cenário implica utilizar ferramentas de educação em saúde para preencher lacunas preexistentes, assim como valorizar a aprendizagem no cotidiano desses sujeitos. Nesse processo, faz-se necessário valorizar ainda as trocas interpessoais permeadas pelo diálogo ${ }^{26}$.

O diálogo poderá ser facilitado para os cuidadores investigados e os idosos porque são, em sua maioria, familiares descritos por laços de consanguinidade. Sabe-se, porém, que nem sempre esse tipo de laço é estabelecido nas relaçóes de cuidado com o idoso e isso exigirá articulação entre saúde e educação, no tocante à formação de profissionais preparados para a inserção no cenário familiar, no qual se inclui a dignidade do cuidado e a potencialização da família para açôes co-participativas em saúde ${ }^{27}$.

As mudanças e a complexidade do envelhecimento apontam para a importância de preparação para o enfrentamento das dificuldades descritas pelos próprios cuidadores durante a tarefa do cuidar. Pode ocorrer a exacerbação ou eclosão de conflitos, provocando estresse e trabalho redobrado ao cuidador. $\mathrm{O}$ cuidador precisa identificar as reais necessidades de saúde dos idosos, garantindo sua autonomia, protagonismo e valorização de sua subjetividade ${ }^{28}$. Essa compreensão do cuidador como sujeito que também necessita e demanda por cuidados deve nortear profissionais de saúde nas açóes dirigidas a eles.

Quanto à aplicabilidade do estudo, os resultados apresentados podem contribuir para o processo, em curso, da construção de uma linha de cuidado aos idosos no contexto estudado, com reflexôes acerca da valorização do cuidador e de suas necessidades educativas, pois “[...] somente será possível cuidar do idoso/família unindo pesquisa e educação. A pesquisa fundamenta as reflexōes e a educação permite compartilhar o conhecimento, resultando na melhoria do processo de cuidar." ${ }^{\text {.26 }}$

As experiências de atenção à saúde, a partir do enfoque das linhas de cuidado, inserem-se como propostas dos serviços para vencer os desafios na produção de cuidado integral, centrada na relação com o usuário, diferente daquela com foco nos procedimentos ${ }^{29}$. Uma vez modificados os processos de trabalho nos serviços de saúde e também a relação desses com a clientela, espera-se que o serviço da atenção primária à saúde, como é o caso da unidade de saúde da família estudada, seja porta de entrada para o sistema e também espaço para construção de vínculos, e que esse espaço entrelaçado aos demais dispositivos comunitários sirvam para a produção de vida com qualidade. 
Nessa perspectiva, é destacável a informação de que, no presente estudo, a maioria dos idosos referiu procurar o hospital em caso de doença, e não a USF, o que pode se constituir em mais um desafio para a estruturação de uma linha de cuidado. É emergente a necessidade de fortalecer os vínculos entre os profissionais da USF e os idosos e seus cuidadores.

As limitaçôes deste estudo referem-se ao pequeno tamanho da amostra e à ausência de dados acerca do grau de dependência e de vulnerabilidade do idoso. Sobre o tamanho da amostra, este impossibilitou identificar aspectos generalizáveis dos resultados apresentados, assim como a ampliação da análise estatística, a exemplo de possível associação existente entre as variáveis qualitativas estudadas. Em relação à ausência de dados acerca do grau de dependência e de vulnerabilidade dos idosos, esses poderiam aprofundar aspectos da capacidade funcional do idoso, do contexto familiar e de seu entorno social, que explicitassem a necessidade do cuidador, assim como as implicaçóes dessas variáveis para a produção de cuidado e para o sentimento de sobrecarga do cuidador.

Pesquisas deste tipo realizadas em outros locais do Brasil, inclusive com amostras maiores, possibilitam a oferta de informaçóes essenciais para o planejamento das intervençôes educativas, tanto no âmbito da saúde do idoso quanto do seu cuidador.

\section{Considerações finais}

Conclui-se que a prática do cuidar direcionada ao idoso conta com a participação voluntária de cuidadores informais e familiares, em sua maioria mulheres adultas e de meia idade, com predominância do nível escolar fundamental incompleto, as quais encontram dificuldades na produção desse cuidado devido à escassez de conhecimentos básicos em saúde, tais como: cuidados com a higiene, boa alimentação, administração de medicamentos, e com a instabilidade emocional do idoso.

Essas dificuldades constituem as necessidades de aprendizagem dos participantes do estudo, que têm interesse educativo e precisam ser atendidos, para que uma intervenção fundamentada na educação em saúde ofereça suporte e alívio da sobrecarga e do estresse relacionados ao desenvolvimento do cuidado ao idoso.

Destarte, o estudo produz subsídios para construção de uma linha de cuidado dirigida a essa população e para realização de uma intervenção educativa contextualizada, participativa e singular no território estudado, permitindo que esse processo educacional seja gerador de crescimento e de mudanças de comportamento na relação entre os profissionais de saúde e os cuidadores e entres estes e os idosos. Conhecer o perfil dos cuidadores e identificar suas necessidades educativas em saúde é imprescindível para consolidação de intervençóes que primem pela saúde da população idosa.

No intuito de alicerçar as linhas de cuidado, uma estratégia fundamental seria pensar no responsável pelo ato de cuidar e incluí-lo em planejamentos e açôes de saúde. Os cuidadores precisam ser treinados, mas, primeiramente, torna-se necessário mapear quais aspectos do conhecimento precisam ser supridos e os tipos de obstáculo a serem removidos.

Portanto, os resultados obtidos no presente estudo podem contribuir para a produção de reflexôes em torno de processos educacionais, humanísticos e éticos, para preencher lacunas de conhecimento e valorizar as habilidades do cuidador, assim como no processo de compartilhamento de saberes e de práticas entre cuidadores e profissionais de saúde.

\section{Referências}

1. Instituto Brasileiro de Geografia e Estatística - IBGE. Projeção da população do Brasil por sexo e idade 1980-2050. Revisão 2008. Acessado no: http://www.ibge.gov.br/home/estatistica/populacao/projecao_da_populacao/2008/

2. Trelha CS, Revaldaves EJ, Yussef SM, Dellaroza MSG, Cabrera MAS, Yamada KN, et al. Caracterização de idosos restritos ao domicílio e seus cuidadores. Espaç Saúde. 2006; 8(1): 20-7. Disponível em: http://www.ccs.uel.br/espacoparasaude/v8n1/v8n1_res_4.htm

3. Vilela ABA, Meira EC, Souza AS, Souza DM, Cardoso IS, Sena ELS, et al. Perfil do famililar cuidador de idoso doente e/ou fragilizado do contexto sociocultural de Jequié-BA. Rev Bras Geriatr Gerontol. 2006; 9(1): 55-69. Disponível em: http://revista.unati.uerj.br/scielo.php?script=sci_ arttext\&pid=S1809-98232006000100005\&lng=pt

4. Reis DT, Eiras DL, Jacóia SC, Silva L, Bousso RS. Quem são os cuidadores de idosos em situação de final de vida atendidos pela Estratégia Saúde da Família? Rev ConScientiae Saúde. 2011; 10(4): 682-8. Disponível em http://www4.uninove.br/ojs/index.php/saude/article/view/2585 
5. Paulo MG, Teixeira AR, Jotz GP, Barba MC, Bergmann RS. Evaluation of quality of life of the caregivers for hearing-impaired elderly: influence of hearing aids use. Arq Int Otorrinolaringol. 2008; 12(1): 24-7. Disponível em: http://www.internationalarchivesent.org/additional/acervo_eng.asp?id=479

6. Meira EC, Gonçalves LHT, Xavier JO. Relatos orais de cuidadores de idosos doentes e fragilizados acerca dos fatores de risco para violência intrafamiliar. Ciênc Cuid Saúde. 2007; 6(2): 171-80. Disponível em: http://periodicos.uem.br/ojs/index.php/CiencCuidSaude/article/view/4148

7. Queiroz ZPV, Lemos NFD, Ramos LR. Fatores potencialmente associados à negligência doméstica entre idosos atendidos em programa de assistência domiciliar. Ciênc Saúde Coletiva. 2010; 15(6): 2815-2824. http://dx.doi.org/10.1590/S1413-81232010000600019

8. Borges DT, Dalmolin BM. Depressão em idosos de uma comunidade assistida pela estratégia de saúde da família em Passo Fundo, RS. Rev Bras Med Fam Comunidade. 2012; 7(23): 7-14. http://dx.doi.org/10.5712/rbmfc7(23)381

9. Brasil. Ministério da Saúde. Portaria GM nº 2.528 de 19 de outubro de 2006 - Política Nacional de Saúde da Pessoa Idosa. Brasília; 2006.

10. Espín AAM. Caracterización psicosocial de cuidadores informales de adultos mayores con demencia. Rev Cubana Salud Pública [online]. 2008; 34(3) [acesso em jan 10]. Disponível em: http://scielo.sld.cu/scielo.php?script=sci_arttext\&pid=S0864-34662008000300008\&lng=es

11. Flores GC, Borges ZN, Denardin-Budó ML, Mattioni FC. Cuidado intergeracional com o idoso: autonomia do idoso e presença do cuidador. Rev Gaúcha Enferm. 2010, 31(3): 467-74. http://dx.doi.org/10.1590/S1983-14472010000300009

12. Fratezi FR, Gutierrez BAO. Cuidador familiar do idoso em cuidados paliativos: o processo de morrer no domicílio. Ciênc Saúde Coletiva. 2011; 16(7): 3241-8. http://dx.doi.org/10.1590/S1413-81232011000800023

13. Paz AA, Santos BRL, Eidt OR. Vulnerabilidade e envelhecimento no contexto da saúde. Acta Paul Enferm. 2006; 19(3): 338-42. http://dx.doi. org/10.1590/S0103-21002006000300014

14. Pimenta GMF, Costa MASMC, Gonçalves LHT, Alvarez AM. Perfil do familiar cuidador de idoso fragilizado em convívio doméstico da grande Região do Porto, Portugal. Rev Esc Enferm USP. 2009; 43(3): 609-14. http://dx.doi.org/10.1590/S0080-62342009000300016

15. Domínguez Guedea MT, Damacena FA, Montiel Carbajal MM, Ochoa Marcobich P, Álvarez Hernández G, Valdéz Lizárraga L, et al. Necessidades de apoio social em cuidadores de familiares idosos mexicanos. Psicol Soc. 2009; 21(2): 242-9. http://dx.doi.org/10.1590/S0102-71822009000200011

16. Fernandes MGM, Garcia TR. Determinantes da tensão do cuidador familiar de idosos dependentes. Rev Bras Enferm. 2009; 62(1): 57-63. http:// dx.doi.org/10.1590/S0034-71672009000100009

17. Sampaio AMO, Rodrigues FN, Pereira VG, Rodrigues SM, Dias CA. Cuidadores de idosos: percepção sobre envelhecimento e sua influência sobre o ato de cuidar. Estud Pesqui Psicol. 2011; 11(2): 590-613. http://www.revispsi.uerj.br/v11n2/artigos/html/v11n2a15.html

18. Camargos EF, Souza AB, Nascimento AS, Morais-e-Silva AC, Quintas JL, Louzada LL, et al. Use of psychotropic medications by caregivers of elderly patients with dementia: is this a sign of caregiver burden? Arq Neuro-Psiquiatr. 2012; 70(3): 169-74. http://dx.doi.org/10.1590/S0004282X2012000300003

19. Pavarini SCI, Tonon FL, Silva JMC, Mediondo MZ, Barham EJ, Filizola CLA. Quem irá empurrar minha cadeira de rodas? A escolha do cuidador familiar do idoso. Rev Eletrônica Enferm. 2006; 8(3): 326-35. [online] [acesso em 15 jan 2013]. Disponível em: http://www.revistas.ufg.br/index.php/ fen/article/view/7071/5002

20. Garbin CASG, Sumida DH, Moimaz SAS, Prado RL, Silva MM. O envelhecimento na perspectiva do cuidador de idosos. Ciênc Saúde Coletiva. 2010; 15(6): 2941-8. http://dx.doi.org/10.1590/S1413-81232010000600032

21. Souza WGA, Pacheco WNS, Martins JJ, Barra DCC, Nascimento ERP. Educação em saúde para leigos no cuidado ao idoso no contexto domiciliar. ACM Arq Catarin Med. 2006; 35(4): 57-63. Disponível em: http://www.acm.org.br/revista/pdf/artigos/395.pdf

22. Fernandes MGM, Garcia TR. Atributos da tensão do cuidador familiar de idosos dependentes. Rev Esc Enferm USP. 2009; 43(4): 818-24. http:// dx.doi.org/10.1590/S0080-62342009000400012

23. Gratao ACM, Vale FAC, Roriz CM, Haas VJ, Lange C, Talmelli LFS, et al. The demands of family caregivers of elderly individuals with dementia. Rev Esc Enferm USP. 2010; 44(4): 873-80. http://dx.doi.org/10.1590/S0080-62342010000400003

24. Rodrigues SLA, Watanabe HAW, Derntl AM. A saúde de idosos que cuidam de idosos. Rev Esc Enferm USP. 2006; 40(4): 493-500. http://dx.doi. org/10.1590/S0080-62342006000400007

25. Organização Mundial da Saúde - OMS. Relatório da Saúde no Mundo. Saúde Mental: Nova concepção, nova esperança. OMS; 2001.

26. Martins JJ, Albuquerque GL, Nascimento ERP, Barra DCC, Souza WGA, Pacheco WNS. Necessidades de educação em saúde dos cuidadores de pessoas idosas no domicílio. Texto Contexto - Enferm. 2007; 16(2): 254-62. http://dx.doi.org/10.1590/S0104-07072007000200007

27. Silva LW, Ribeiro FB, Amaral RL, Ferreira RS, Souza TF. A família e o cuidado - reveses e vieses entre a aceitação e o desafio de cuidar. Rev Kairós Gerontol. 2010; 13(2): 191-202. Disponível em: http://revistas.pucsp.br/index.php/kairos/article/view/5375

28. Martins JJ, Borges M, Silva RM, Nascimento RPE. O processo de viver e de ser cuidado de idosos e a percepção dos cuidadores. Rev Cogitare Enferm. 2011; 16(1): 96-103. Disponível em: http://ojs.c3sl.ufpr.br/ojs2/index.php/cogitare/article/view/21118

29. Franco TB. Integralidade na assistência à saúde: a organização das linhas do cuidado. In: Merhy EE, Magalhães HM Jr, Rimoli R, Franco TB, Bueno WS. O trabalho em saúde: olhado e experienciando o SUS no cotidiano. São Paulo: Hucitec; 2004. p.125-134. 